\title{
Vorwort des Verfassers
}

Non mihi res sed me subiungere rebus.

Ulrich von Wilamowitz-Moellendorffs „Die llias und Homer" hat mir, was ich ahnte, zur Gewißheit werden lassen: in der Ilias müsse das Urgedicht Homers enthalten sein; diesem müsse jene, was sie an Einheit habe, verdanken. Man mag. wie Wilamowitz die Homerforschung, einer Ausgrabung, also die Ilias etwa dem unausgegrabenen Hügel von Hissarlik vergleichen; doch die kritische Forschung. nach Homer und seiner Dichtung ist grundverschieden von dem Suchen des Spatenforschers nach der Stadt des Priamos oder irgendeiner anderen verschütteten Stätte. Sie wäre selbst dann noch verschieden, wenn solcher Schutthügel durchsichtig wäre, wie es die Ilias ja.ist; wenn seine sämtlichen Teile, unterste wie oberste, älteste wie jüngste, dem Ausgräber, bevor er den Spaten ansetzte, ebenso gleichmäßig vor Augen lägen, alle auch gleichzeitig zugänglich wären, wie dem Homerforscher vom Anfang bis zum Ende seiner, Arbeit alle Teile der Ilias. Denn Troja so gut wie Pompeji, Athens Akropolis wie Roms Forum haben uns gelehrt den zweiten Unterschied, daß jede Zeit dem reellen und praktischen Zweck eigenes Werkes und Bedürfnisses das Werk früherer Zeiten opfert, zerstörend oder ändernd, soweit eigene Zwecke es fordern. Wie könnten aber spätere Dichter in solcher Weise verfahren haben auf rein id e ellem Gebiet mit dem großen Werk, dessen Bewunderung sie zur Nachfolge und Weiterdichtung reizte? Was ohne Wissen und Willen mündliche Überlieferung von Generation zu Generation am Wortlaut der Dichtung än- 
derte, ist jener bewußten, gewalttätigen Änderung selbst geheiligter Bauwerke nicht zu vergleichen. Kann demnach eine methodische Ausgrabung, zumal wo kein Pausanias sie leitet, nur von den obersten, spätesten Schichten beginnend, vorsichtig in die Tiefe dringen, so kann der Homerforscher sogleich nach dem Kerne greifen. Sein Weg ist der entgegengesetzte des Ausgräbers: ihm ist der Homer, den er sucht, selbst Führer. Muß doch dessen Gold von dem. Elektron, dem Silber, dem Kupfer der Späteren für den, der ohne vorgefaßte Meinung sich der Sache hingibt, am Pruifstein der Kritik selbst sich scheiden.

Als das Zorngedicht Homers so sich überraschend aus der Masse der Ilias heraushob und das zeitliche Kunstwerk auch wie ein räumlich es vor Augen lag, da wurde zu dem Rhythmus des Ganzen auch die Symmetrie seiner fünf Teile offenbar:

hier I, der Anfang, der stolze König und der edelste Held, angesíchts der Feinde, in leidenschaftlichem Streite entzweit, aus der Ilias $\boldsymbol{A}$.

dort $V$, das Ende: versöhnender Abschluß über den Gräbern des Patroklos und Hektors, deren Tod und Grab schon auch Achilleus' Tod und $\mathrm{Grabmal}$ bedeuten, aus der Ilias $\Omega$.

Dazwischen drei Akte, in denen das große Geschick des Achilleus sich erfüllt :

Der erste $I I$ I. Agamemnons Aufgebot; aus $B$; 2. die erste Niederlage, aus $\Gamma \Delta E[Z H] \Theta ; 3$; das abgewiesene Sühneangebot, aus $\boldsymbol{I}$;

der zweite $I I I$ I. die zweite Niederlage, aus $[K] \Lambda$; 2 . die Wendung, da, durch Patroklos' Flehen gerührt, Achill den Freund zur Rettung der Schiffe aussendet, aus $A M[N \Xi] O \Pi ; 3$. Patroklos' Sieg und Tod, aus $\Pi P$; der dritte IV I. Achills Schmerz und Reue, seine Zornabsage, um Patroklos an Hektor zu rächen, aus $\Sigma T$; 2. Achilis Rache und Hektors Tod, aus TY $\boldsymbol{\Phi X}$; 3. Patroklos' Bestattung, seine Aschenurne und sein 
angefangener Grabhügel, bestimmt, bald auch Achills Asche zu umschließen, aus $\Psi$.

Also dreimal drei Teile'von annähernd gleichem Umfang, doch ohne das Blendwerk gleicher Verszahlen. Eine Symmetrie des Aufbaus, die, weil ungèsucht sich ergeḅend, als erste Bestätigung wohl gelten durfte.

Hinzu kam die zweite. Erst nachdem Homers Gedicht die übrige. Ilias von sich abgeschüttelt hatte, las ich Erich Bethes Homer, danach auch die fleißigen Einleitungen von Ameis-Hentze zu den vierundzwanzig Gesängen der Ilias, sowie W. Leafs Introductions. Da fand ich sehr viele, vielleicht die meisten meiner Ausscheidungen bereits von andern gemacht, freilich, auch von Bethe, nicht wenig des Echtesten und Besten verworfen und die Einheit da gefaßt, wo sie am wenigsten vorhanden, in der Ilias als solcher.

Den griechischen Text Homers zu bieten, will die Ungunst der Zeit nicht verstatten, ich muß mich einstweilen begnügen, die Zahlen der Verse anzugeben, welche die fünf Teile des Zorngedichts bilden; mit den wichtigeren Änderungen, die an August Naucks Text, Homeri Ilias cum potiore lectionis varietate edidit Augustus Nauck. Berolini apud Weidmannos (1877 und 1899), nötig wurden. Wo zwei Verse durch t- verbunden .werden, ist die Verbindung 'meistens ohne weiteres klar. Geringere Textänderungen sind übergangen.

I.

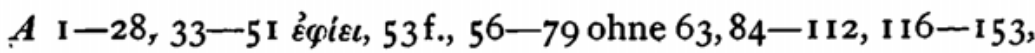

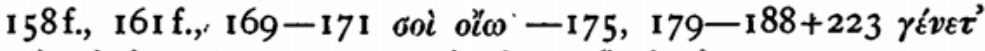

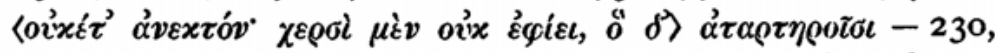
234f., 240-26r, 273-279, 285-30r ohne 296 und 294

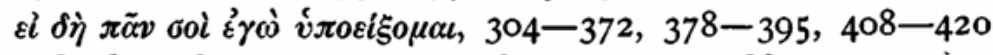
428, danach 497-531 + 430f., 472-474, 488-492.

II.

B 48-66 (nach $50 A$ I02 wiederholt), 72-93 + 100f., 109-,

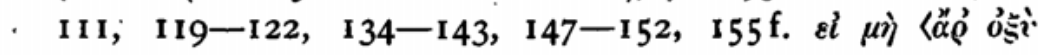




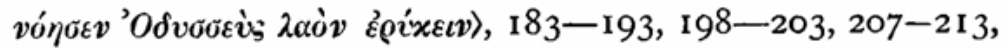

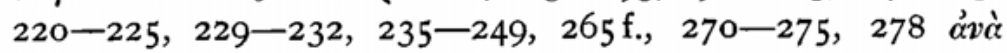

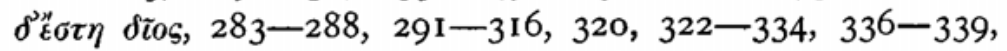
$34 \mathrm{I}-347,369-376,379-38 \mathrm{I}, 394$ f., 398-40I, 459-466,

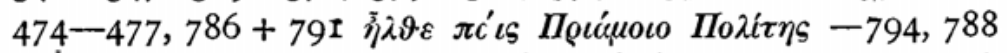

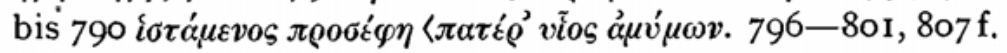

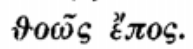

$\Gamma$ If., 8 -I 4 .

$4446-45 \mathrm{I}$.

$\boldsymbol{E} 519-523,528-530,533-542,550-553,561-566,568-$ 586, 590 f., $608-618,620-626$.

$\theta$ 66-I I 3 ohne 73 f., I I 5-I22, I 32-I 42, I 57-I 59, 336-349, $485-505,507-516,542-545,547,553-556,560 f$.

I I-3, 89-100, 103-124, I28-138, I62-205, 192 Фoiv $t \xi$ S'if 223-235, 247-250, 260-266, 270-280, 300-319, 344347, 378-38o, 356-36I, 417-424, 427-443, 496-498, $515-523,600-603,606-608,611-614,617-637,640$ $-657,669-681,693,695-700,704-705+706-713$.

III.

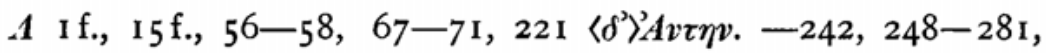
$292-298,30 \mathrm{I}-306,3 \mathrm{II}-325,369-372, .376-38 \mathrm{I}, 384$

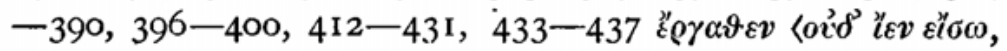
$439,447-469,472-475,482-49 \mathrm{I}, 504-514,5 \mathrm{I} 6-520$, $55^{8}-568,575-603,608-623,64 \dot{4}-652,655-668+790 \mathrm{f}$., $804-811,814-830,837-848$.

II I-4, 265-267, 269 + 27I-280, 287-289, 430-432, 437 $-449,45 \mathrm{I}-462,467-47 \mathrm{I}$.

O 390-407, 415-421, 674-678, 704, 717f., 726-731.

II I-7, 12, 17-39, 46-54, 60-65, $64 \mu \dot{\dot{t} v} \alpha^{\prime} \gamma \dot{\alpha} x \lambda v \tau \alpha, 8 \mathrm{I}-83$, $87-92,95$ f., IоI f., I1 4 " $E x \tau \omega \varrho \delta^{\prime}-$ I 30, I 55 , I99 $\sigma \tau \eta \tilde{\sigma \varepsilon} \delta^{\prime}$, $22 \mathrm{I} \beta \tilde{\eta} \delta^{\circ}, 225-24 \mathrm{I}, 246 \mathrm{f}$., 249-26o, 262, 266f., 278, 280 -282, 284f., 287-290, 293-296, 364-368, 394-398, 419-426, 428-430, 476"'Ev $9^{\prime \prime} \alpha \alpha-486,783-788,79$ I f., 805 $-807,8$ r 3 f., 816-821, 829, 837-845, 847-849, 85 I-863. 
p 125-133, I38f., 262-267, 274-280, 288-30o ohne 296, 304-318, 384-397, 593-6oo, 603f., 6orf., 605-6o8

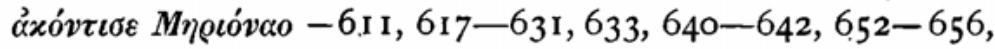
$682-697,706-708,712-734$.

IV.

S I-8, 15-27, 70-8I, 88-II6 ohne 106, I26-I29, I38,

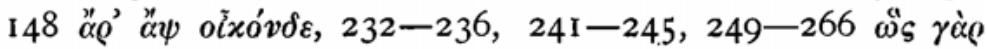

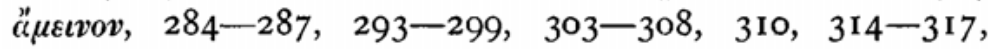
$324-328,333-335,343-355$.

I If., 40-42, 45-64, 67-70, 74-76, 78, 85-90, 137-148, 276-279, 357-364.

$Y$ 3, 38I-388+395-400, $407-4 \mathrm{I} 8,490-494$.

$\Phi$ I $-5,6+7$ f., 34 für $\Delta \alpha \varrho \delta \alpha v i \delta \alpha o$ vielmehr $\delta i o s ~ ' A \chi \imath \lambda \lambda \varepsilon \dot{r}^{\prime} \varsigma$ - 36, 40, 44-49, 53-59, 64-72, 74f., 97-107, I I4-I19, 205 f., 209-212, 214, 216-227, 540-543, 606f.

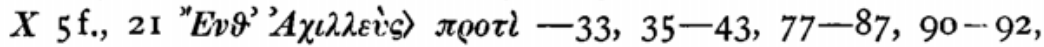
98-I10, I3I-I65 ohne I52, 208-2I2, 225, 248-262, 264-269, 273-275, 278, 283-285, 289-293+306 ov dzे

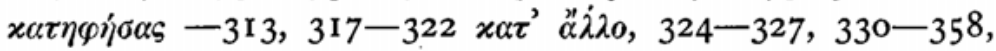
$36 \mathrm{I}-370,372-374,376,396-402,405-419,429+437$,

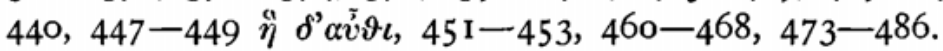

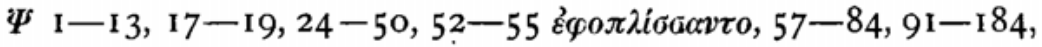
192-195, 197, 214-256.

$\mathrm{V}$.

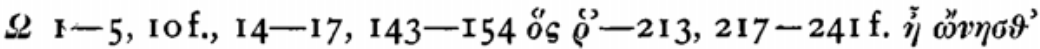

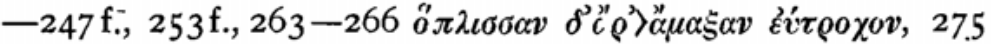

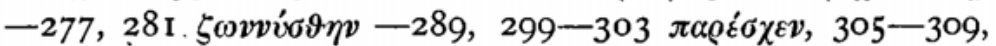

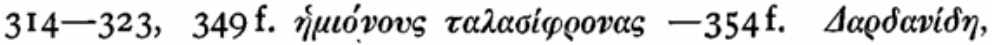

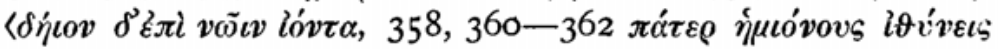

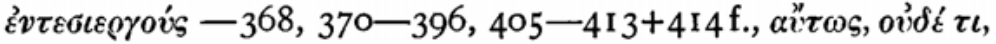

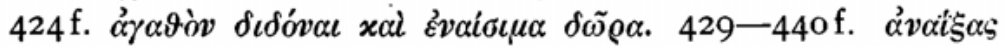

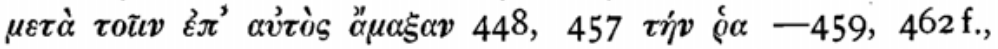

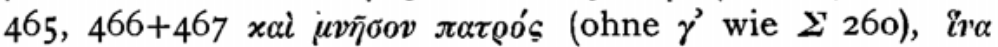

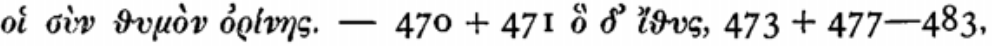
$485-493,498-513,5 I_{5}-524,552-555,559-56 \mathrm{I}+563 \mathrm{f}$. 


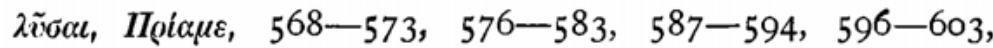

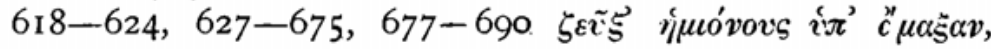

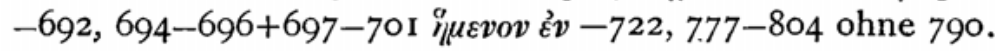

Die Ausscheidung hat sich von selbst gemacht; aber danach mußte doch das Nichthomerische auf seine eigene Art und seinen Stil angesehen werden; nicht allein mit dem Homerischen, sondern die verschiedenen Zutaten auch miteinander verglichen werden, sowohl zu weiterer Prüfung jener Ausscheidung als zur Erkenntnis der Entwicklung, die die epischè Poesie nach Homer durchgemacht hat. Dieser Vergleichung widmet sich diese Untersuchung. Sie vollzieht sich in folgenden vier Stücken:

I. Die Einheitlichkeit des Gedichts vom Zorn des Achilleus.

2. Die Homeridendichtungen einzeln betrachtet.

3. Art und Stil dieser Homeridendichtungen im ganzen betrachtet.

4. Mythologie und Religion bei Homer und bei den Homeriden.

Von persönlicher Kritik und Polemik ist abgesehen.

$\mathrm{H}$ a mburg.

EUGEN PETERSEN. 